\title{
A Stackelberg Game Approach for Energy Outage-Aware Power Distribution of an Off-Grid Base Station over Multiple Retailers
}

\author{
Seung Hyun Jeon ${ }^{1}$, Joohyung Lee ${ }^{2, *}$ and Hong-Shik Park ${ }^{1}$ \\ 1 School of Electrical Engineering, the Korea Advanced Institute of Science and Technology, \\ Daejeon 34141, Korea; creemur@kaist.ac.kr (S.H.J.); park1507@kaist.ac.kr (H.-S.P.) \\ 2 Department of Software, Gachon University, Seongnam 13120, Korea \\ * Correspondence: j17.lee@gachon.ac.kr
}

\begin{abstract}
This paper investigates the problem of power distribution for an off-grid base station (BS) that operates sustainably without an electrical grid. We consider that multiple retailers with heterogeneous renewable energy sources (RESs) compete to maximize their revenues by individually setting the unit power price. Energy outages (EOs), which cause the power supply to fall below that which is sufficient for ensuring the traffic arrival rate required for the off-grid BS, critically affect the users' service quality. To minimize EOs and operational expenditure (OPEX), the off-grid BS manages the power supply by reacting to the retailers' pricing decisions. We analyze the economic benefits of power distribution to the off-grid BS from the perspective of the retailers' pricing competition, by designing a hierarchical decision-making scheme as a multi-leader single-follower Stackelberg game. We derive a closed form expression for the optimal behavior of the off-grid BS and retailers, based on well-designed utility functions. Finally, numerical results demonstrate the proposed solution with its practical convergence time.
\end{abstract}

Keywords: off-grid base station (BS); multiple retailers; energy outage (EO); power distribution; unit power price; multi-leader single-follower Stackelberg game

\section{Introduction}

For cost-effective deployment of BSs over wide areas with scattered population, off-grid base stations (BSs) are receiving significant attention. This is because such off-grid BSs can be installed in a standalone manner, not requiring macro-grids. Recently, the off-grid BSs have been further developed, driven by the desire to utilize renewable energy sources (RESs) without diesel generators (DGs). This approach aims to address growing concerns of environmental damage and increased operational expenditure (OPEX) associated with DGs, in places where DGs have become the standard source of power for off-grid sites [1]. However, owing to highly variable, unpredictable, and intermittent generation of RESs [2,3], reliable operation of off-grid BSs remains a challenging issue. These off-grid BSs are likely to be subject to energy outages (EOs) when they cannot satisfy the their power demand to ensure satisfactory service quality for mobile users [4-6].

To design RES based power distribution mechanisms for BSs, different power distribution problems have been addressed in the literature, aiming to reduce greenhouse gas (GHG) emissions and/or the cost of energy procurement, where sufficient power support was considered $[7,8]$. Recently, a novel EO-aware power distribution scheme (EO-PDS) for minimizing EOs of mobile operators under the condition of insufficient RESs was proposed [6]. However, because that proposal assumed a single off-grid BS with the RESs' harvester in an arbitrary area, no consideration was given 
to the unit power price as an optimization factor, which limits the applicability and feasibility of the proposed model. For off-grid sites, multiple retailers may exist, and their pricing competition may strongly affect power distribution mechanisms to off-grid BSs.

In this paper, we investigate the problem of power distribution for an off-grid BS where multiple retailers compete to maximize their revenues and where EOs may occur owing to insufficient energy supply by RESs. We consider that retailers with heterogeneous RESs individually set their unit power prices. Then, the off-grid BS aiming to minimize EOs and OPEX reacts to the retailers' pricing decisions for deciding on the optimal power distribution, considering the traffic arrival rate to the off-grid BS. Accordingly, we consider the iterative interaction between the competing retailers and the off-grid BS when designing an optimal power distribution scheme (PDS) as well as an optimal pricing decision scheme. This is achieved by designing a hierarchical decision-making scheme as a multi-leader single-follower Stackelberg game. For this, we design well-defined utilities and then derive a closed form expression for the optimal unit power price and the optimal power distribution. Using the analysis of economic benefits, we demonstrate that the proposed approach yields a unique equilibrium solution and provides an acceptable convergence time. The main contributions of this paper are summarized as follows:

- We investigate EO-aware power distribution based on a Stackelberg game analytical framework with multiple retailers and an off-grid BS under the sufficient and insufficient RES's generation. The proposed game-theoretic approach based power distribution is then decomposed into two sub-problems, which include the power distribution and the pricing decision.

- We formulate the power distribution problem in the off-grid BS operation as a multi-leader single-follower Stackelberg game, where the well-defined utilities are introduced to maximize the retailers' revenues and to minimize EOs and power cost of the off-grid BS. The Stackelberg equilibrium (SE) of two sub-problems is obtained based on the Lagrangian dual function and gradient descent method.

- The Stackelberg game based power distribution can guarantee the retailers' revenues under providing unreliable power supply to the off-grid BS. Under such circumstances, the off-grid BS can reduce EOs as well as OPEX efficiently. Based on iterative power distribution and pricing decision algorithms, the proposed solution can converge to a unique equilibrium within an acceptable convergence time.

The remainder of the paper is organized as follows: Section 2 introduces the recent literature relating to the off-grid BS, power distribution, EOs, and game-theoretic approaches. In Section 3, we propose a Stackelberg game based PDS and formulate a retailer stage model and an off-grid BS stage model. In Section 4, the solutions of the proposed multi-leader single-follower Stackelberg game are presented, and the optimal power distribution and the optimal unit power price are obtained, using the proposed power distribution and pricing decision algorithm. Through a rigorous game-theoretic approach, we analyze the performance of the proposed power distribution and then show our approaches converge to a unique equilibrium in Section 5. Finally, we conclude our paper in Section 6.

\section{Related Work}

A non-depletable and non-polluting RES features an alternative energy source [9]. For remote sites in developing countries gird connected systems are not allowable due to high installation cost. In order to reduce OPEX and improve reliability against RES's intermittent power supply, various power generators are required to be integrated. According to the recent researches [10,11], off-grid BSs in such remote sites are predicted to deploy almost 400,000 and grow 22 percent by the year 2020 in the world. Various BS models for remote off-grid sites are introduced, including the primary and backup solutions such as RESs, DGs, and battery storage [12]. Since DGs with low capability and efficiency induce environmental problems as well, DGs as backup will be substituted into battery storage like Photovoltaic (PV)-Wind systems. In [13], the authors discussed a zero-grid BS system, which solely 
relies on RESs. In [14], the authors proposed a price based multiple PDS for off-grid BSs with batteries powered by multiple RES retailers. However, the multiple PDS does not consider the existence of EOs. Since the normalized energy profiles for short and long time terms are different in [15], solar and wind energy can be proper off-grid solutions to power small BSs such as micro and femto. In [16], PV-Wind systems for off-grid BSs in South Korea reduce OPEX more than DGs. Nevertheless, battery solutions for the off-grid BS operation are required. In general, EOs can occur when RES's generation are not sufficient or off-grid BS's battery is empty [4,5]. EOs mean that the shortage of power supplies does not guarantee service quality to mobile users. Thus, EOs are one of challenging issues for the reliable off-grid BS operation.

Recently, some researches on EOs of off-grid BSs have been studied. The authors of [17] first proposed an analytic framework to evaluate a power outage probability of a solar powered BS. For given a tolerable power outage probability, they obtain the cost-optimal PV panel and battery dimensions. In [18], the authors proposed EO-aware cell association to periodically broadcast battery levels of the energy harvesting BS. Besides, the authors of [19] proposed a green energy, delay-aware user association, and resource allocation scheme for off-grid solar powered BSs. Their proposed temporal energy allocation algorithm only considers solar PV and battery solutions as primary supply of green energy budget. However, lack of generation (i.e., EOs) was not considered. Moreover, it is difficult to easily understand that amount of the excess energy for the expected battery level is discarded. The remaining energy can prevent the unexpected future EOs. The work of [6] firstly proposed an EO-PDS for minimizing EOs of mobile operators where insufficient RESs were considered. However, since they assume a single retailer based off-grid BS model in an arbitrary area, there was no consideration of unit power price that affects to choose which RES distributes to the off-grid BS. Thus, the pricing competition between multiple retailers highly impacts on power distribution mechanism for the reliable off-grid BS operation.

Next, a few researches on game-theoretic approaches have been introduced. The authors of [20] proposed a cooperative Nash bargaining game based power allocation framework between a primary macro BS and cognitive secondary small BSs. However, due to the sufficient conventional electricity generation, the power distribution problem of the retailers that generate RESs is not considered to cognitive small BSs. On the other hand, the authors of [21] proposed a non-cooperation game based power allocation mechanism for energy harvesting enabled small BSs. Nevertheless, these small BSs connecting to the power grid are considered. Thus, any circumstance of EOs does not happen.

\section{Game Formulation}

\subsection{System Model}

We assume that a BS can be powered using the retailers' RESs at off-grid sites. The retailers, which individually own various RESs such as solar PV panels and wind turbines, reliably provide power $p_{1}, \cdots, p_{k}$ to the off-grid BS and increase their profits. Let $K$ denote the set of retailers, $K \in\{1,2, \cdots, k\}$. When the power supply of the retailers is insufficient, the power distribution to support the BS's traffic is not satisfactory. In this situation, at least one user within BS's coverage area experiences an EO. For reliable power distribution to the off-grid BS, we formulate the unit power price of retailers (multi-leaders) and the power distribution of the off-grid BS (a single follower) as a two-stage Stackelberg game. During the first stage, a $k$-th retailer reports its unit power price $\xi_{k}$ to the off-grid BS. In the next stage, the off-grid BS requests the power distribution $p_{k}$ to the $k$-th retailer, based on the provided unit power price. By iterative cooperation, both the retailers and the off-grid BS can obtain the optimal unit power price and the optimal power distribution, respectively. As shown in Figure 1, the mobile traffic model to the off-grid BS follows the downlink packet arrival [6]. We assume that the mean arrival rate of the off-gird BS $\lambda$ in each fixed time interval is uniformly distributed over $\left[\lambda_{\min }, \lambda_{\max }\right]$. The mean throughput $R$ of the off-grid BS is defined as $R=\lambda \delta$, where $\delta$ is a constant file size. 


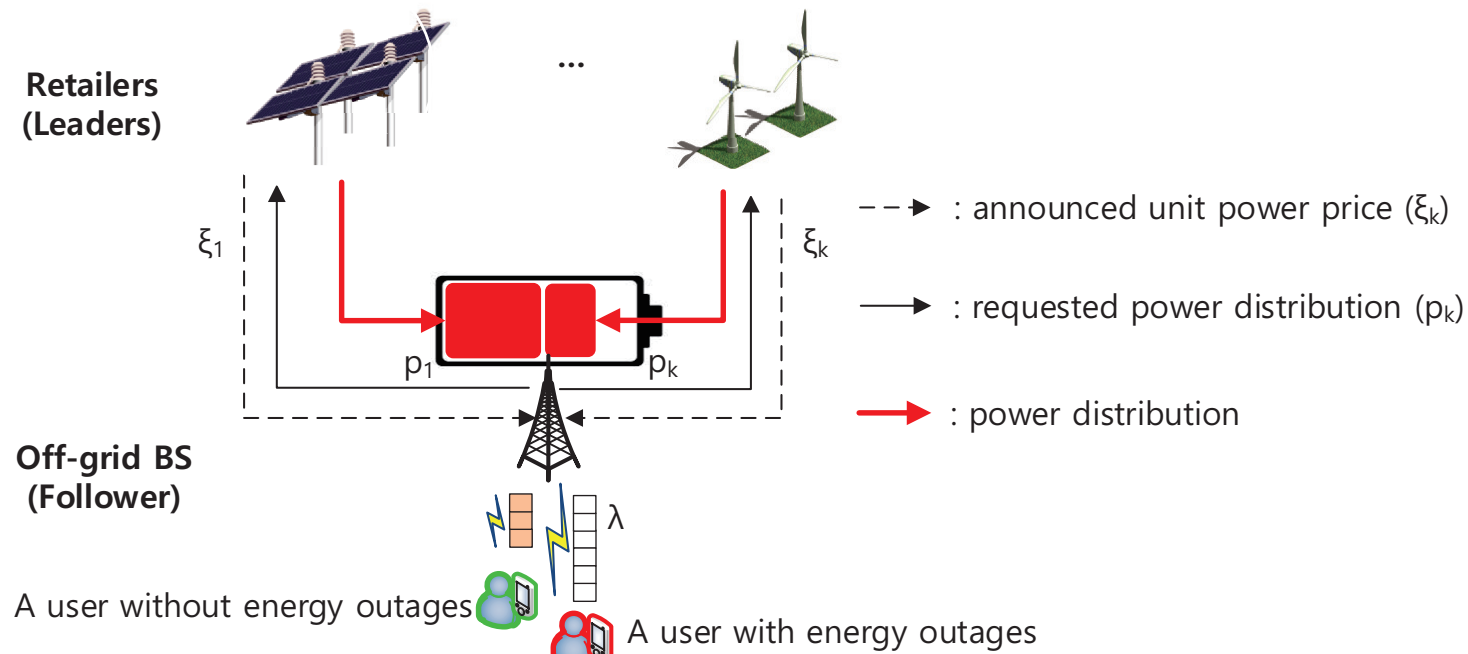

Figure 1. Stackelberg game based power distribution.

In general, for the mean arrival rate $\lambda$, the operational power required by a BS features a fixed power consumption $P_{f i x}$ as the maximum power budget of the BS supplied from the conventional power grid, a fraction for BS's constant power consumption $q$, and a maximal bandwidth capacity $C[6,22]$ as follows:

$$
P_{r e q}^{o p}(\lambda)=q P^{f i x}+(1-q) \frac{P^{f i x} \delta}{C} \lambda
$$

Here, the first and second terms are the constant and variable power consumption of a BS, respectively. Moreover, $\delta \lambda / C$ of the second term is defined as BS load [22]. Next, the BS's transmission power consumption can be understood as follows [23]: $\Delta_{p}$ is the slope of the load-dependent power consumption, $N_{t r x}$ is the number of transceiver chains, and $P_{0}$ is the BS's power consumption at the minimal non-zero output power, respectively. On the off-grid BS side, using Equation (1) and [23], the required transmission power relating to the mean arrival rate $\lambda[6]$ is derived as follows:

$$
P_{r e q}^{t x}(\lambda)=\frac{1}{\Delta_{p}}\left(\frac{P_{r e q}^{o p}(\lambda)}{N_{t r x}}-P_{0}\right)=\alpha \lambda+\beta,
$$

where $\alpha=(1-q) P^{f i x} \delta / \Delta_{p} N_{t r x} C$ and $\beta=\left(q P^{f i x}-N_{t r x} P_{0}\right) / \Delta_{p} N_{t r x}$. Equation (2) as the required transmission power of the off-grid BS can describe as a linear function for the mean arrival rate $\lambda$.

On the retailers' side, corresponding to the required operational power $P_{r e q}^{o p}(\lambda)$ the aggregated power distribution can be expressed as $P_{a g g}(\mathbf{p})=\sum_{k=1}^{K} p_{k}$. Here, $\mathbf{p}$ is the power distribution vector. Thus, as in Equation (2) we can expect the available transmission power from the aggregated power distribution to be given by

$$
P_{a g g}^{t x}\left(P_{a g g}(\mathbf{p})\right)=\frac{1}{\Delta_{p}}\left(\frac{P_{a g g}(\mathbf{p})}{N_{\text {trx }}}-P_{0}\right),
$$

where $P_{a g g}^{t x}\left(P_{a g g}(\mathbf{p})\right)$ ranges from 0 to a maximal value. Considering a macro BS, the maximal transmission power is defined as $20 \mathrm{~W}$.

Let us compare the required transmission power in Equation (2) with the available transmission power from the aggregated power distribution, given by Equation (3). When the available transmission power is lower than the required transmission power that reflects the BS's demand, EOs occur. 
Thus, using [6] we can derive the EO probability for the aggregated power distribution of the off-grid BS, as follows:

$$
\begin{aligned}
p_{e o}\left(P_{\text {agg }}(\mathbf{p})\right) & =\mathbb{P}\left\{P_{\text {req }}^{t x}(\lambda)>P_{\text {agg }}^{t x}\left(P_{\text {agg }}(\mathbf{p})\right)\right\} \\
& =1-\frac{P_{\text {agg }}^{t x}\left(P_{\text {agg }}(\mathbf{p})\right)-\left(\alpha \lambda_{\min }+\beta\right)}{\alpha\left(\lambda_{\max }-\lambda_{\min }\right)} .
\end{aligned}
$$

\subsection{Retailer Stage Model}

As multiple leaders, the retailers seek to maximize their utilities by charging the unit power price to the off-grid BS, and to improve their revenues for the power distribution. The utility function of the $k$-th retailer capturing the revenue is given by

$$
u_{k, R}(\boldsymbol{\xi}, \mathbf{p})=\xi_{k} p_{k}
$$

where $p_{k}$ is the power distribution of the $k$-th retailer and $\xi_{k}$ is the unit power price of the $k$-th retailer's power distribution. Hence, the optimization problem of maximizing the revenues of the retailers [24] can be formulated as

$$
\begin{gathered}
\max _{\boldsymbol{\xi} \geq 0} \sum_{k \in K} u_{k, R}(\boldsymbol{\xi}, \mathbf{p}) \\
\text { s.t. } p_{k} \leq P_{k}^{g e n},
\end{gathered}
$$

where $P_{k}^{g e n}$ is the amount of power generated by the $k$-th retailer's RES. Here, the power distribution $p_{k}$ is constrained by the $k$-th retailer's power generation.

\subsection{Off-Grid BS Stage Model}

As a single follower, the off-grid BS seeks to maximize its utility from the power distribution by the retailers, while reducing the occurrence of EOs and the power cost. Based on [6], the utility function of the off-grid BS is given by

$$
U_{B S}(\mathbf{p})=\ln \left(1+\eta_{1} \frac{P_{a g g}(\mathbf{p})}{P_{r e q}^{o p}\left(P_{r e q}^{t x}(\lambda)\right)}\right)-\eta_{2} p_{e o}\left(P_{a g g}(\mathbf{p})\right)-\sum_{k=1}^{K} \xi_{k} p_{k}
$$

where $\eta_{1}$ and $\eta_{2}$ are weighting factors for the satisfaction of power distribution and penalty of EOs, respectively. Accordingly, the first term means that according to the power demand of the off-grid BS the satisfaction of power distribution is represented. Next, the second term describes the penalty of insufficient power supplies by EOs. Last, $\sum_{k=1}^{K} \xi_{k} p_{k}$ as the third term is the power cost for power distribution from retailers. Hence, the optimization problem [24] for maximization of satisfaction from power distribution and for minimization of EOs and power cost of the off-grid BS can be defined as

$$
\begin{gathered}
\max _{\mathbf{p} \geq 0} U_{B S}(\mathbf{p}) \\
\text { s.t.P } P_{\text {agg }}(\mathbf{p}) \leq P_{\text {req }}^{o p}\left(P_{\text {req }}^{t x}(\lambda)\right) .
\end{gathered}
$$

The aggregated power distribution $P_{a g g}(\mathbf{p})$ is constrained by the BS's power demand for the BS's traffic arrival $P_{r e q}^{o p}\left(P_{r e q}^{t x}(\lambda)\right)[6]$, where the expected BS's power demand can be obtained from Equations (2) and (3).

\section{Solution of the Proposed Stackelberg Game}

The goal of the proposed Stackelberg game is to find the SE by deviating from which neither the retailers nor the off-grid BS will derive any benefits. Because the follower's strategy in the first stage 
will affect the leaders' strategies in the second stage, we employ backward induction to derive a closed form expression [24,25].

\subsection{Optimization of Power Distribution for the Off-Grid BS}

Because the transformed problem in Equation (9) is a convex problem as $\min _{\mathbf{p} \geq 0}-U_{B S}(\mathbf{p})$, it can be solved by dual decomposition [26]. The Lagrangian function [24] can be derived as

$$
L(\mathbf{p}, \boldsymbol{\mu})=\ln \left(1+\eta_{1} \frac{P_{\text {agg }}(\mathbf{p})}{P_{a g g}\left(P_{r e q}^{t x}(\lambda)\right)}\right)-\eta_{2} p_{e o}\left(P_{a g g}(\mathbf{p})\right)-\sum_{k=1}^{K} \xi_{k} p_{k}-\boldsymbol{\mu}\left\{P_{a g g}(\mathbf{p})-P_{a g g}\left(P_{r e q}^{t x}(\lambda)\right)\right\},
$$

where $\boldsymbol{\mu}$ is the nonnegative dual variable corresponding to the constraint in Equation (10) where $\boldsymbol{\mu}=\left(\mu_{k}: k \in \mathrm{K}\right)$ is a vector of Lagrange multipliers [24]. The dual function [24] can be expressed by

$$
g(\boldsymbol{\mu})=\max _{\mathbf{p} \geq 0, \forall k} L(\mathbf{p}, \boldsymbol{\mu}) .
$$

The dual problem corresponding to the primal problem in Equation (9) [24] can be defined as

$$
\min _{\boldsymbol{\mu} \geq 0, \forall k} g(\boldsymbol{\mu})
$$

Because the primal problem in Equation (9) is a convex optimization problem, a strong duality exists [24]. Thus, the dual function can be decomposed into a sub-problem for power distribution. From the Karush-Kuhn-Tucker (KKT) conditions [24] stating that $\partial g(\boldsymbol{\mu}) / \partial p_{k}=0$, the optimal power distribution strategy for the off-grid BS is derived as follows:

$$
p_{k}^{*}=\frac{1}{\eta_{1}}\left\{\frac{\Delta_{p} N_{t r x} \alpha\left(\lambda_{\max }-\lambda_{\min }\right)}{\Delta_{p} N_{t r x}\left(\xi_{k}+\boldsymbol{\mu}\right) \alpha\left(\lambda_{\max }-\lambda_{\min }\right)-\eta_{2}}\right\}-\frac{1}{\eta_{1}} P_{a g g}\left(P_{r e q}^{t x}(\lambda)\right)-\sum_{j \neq k}^{K} p_{j},
$$

where $p_{k}^{*} \geq 0$. In this case, Equation (14) captures the fact that retailers can cooperate to meet the BS's power demand, and according to the unit power price $\xi_{k}$, dual variable $\boldsymbol{\mu}$, and other retailers' power distribution $\sum_{j \neq k}^{K} p_{j}$ including the above-mentioned parameters, the optimal power distribution for the off-grid BS is decided. Here, power distribution of the other retailers refers in Algorithm 1.

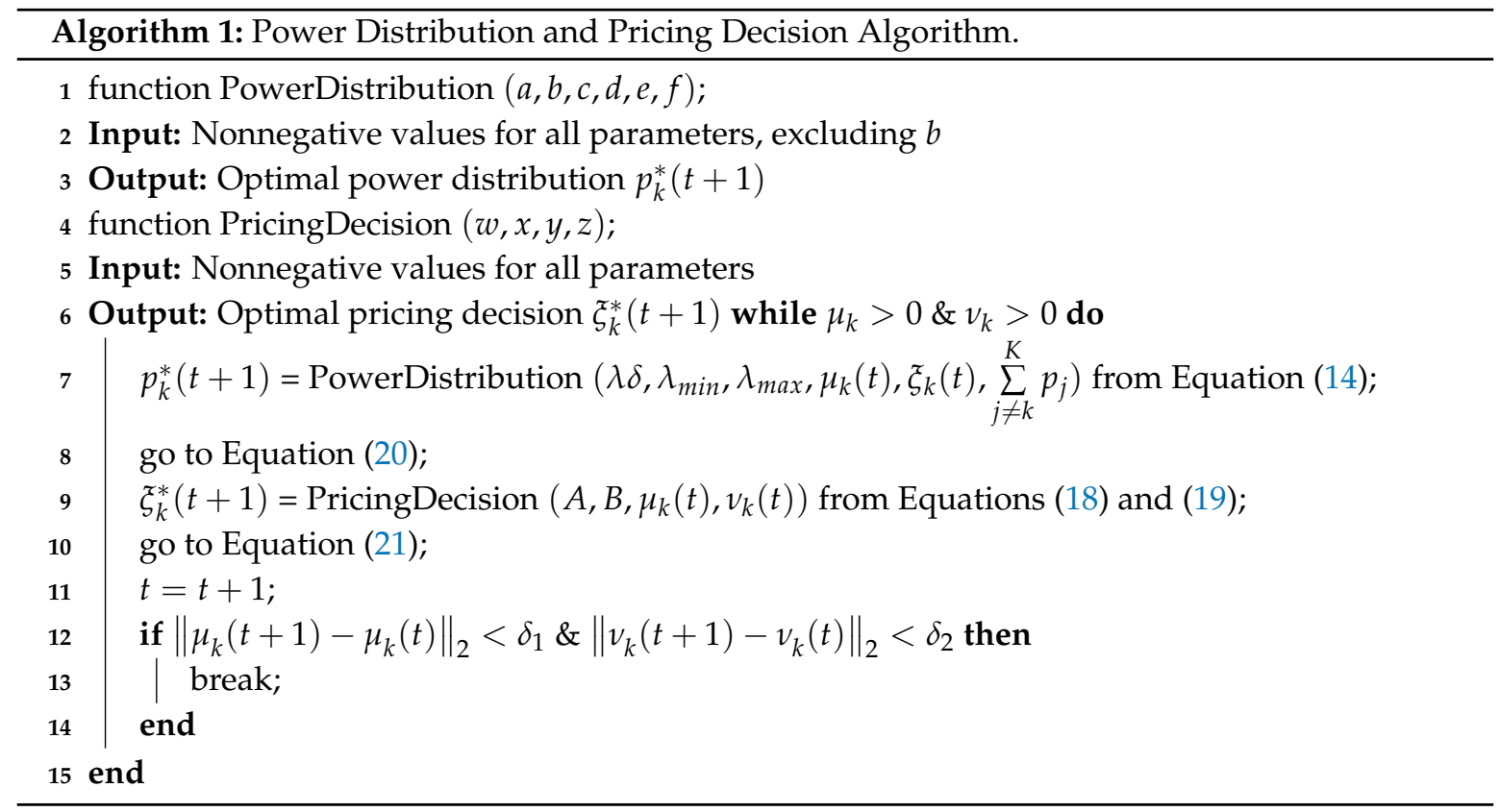




\subsection{Optimization of Unit Power Price for the Retailers}

To maximize the retailer's utility, the $k$-th retailer will adaptively decide the unit power price $\xi_{k}$ based on the power distribution of the off-grid BS. Here, we decompose Equation (6) into a sub-problem: find $\xi_{k}$ to achieve the optimal power distribution of the $k$-th retailer $p_{k}^{*}$, and then decide the optimal unit power price $\xi_{k}^{*}$. Thus, we can solve the unit power price problem using backward induction and dual decomposition, and the Lagrangian function can be derived as follows:

$$
L(\boldsymbol{\xi}, \boldsymbol{v})=\xi_{k} p_{k}^{*}-v_{k}\left(p_{k}^{*}-P_{k}^{g e n}\right),
$$

where $\boldsymbol{v}$ is a nonnegative dual variable corresponding to the constraint of Equation (7) where $\boldsymbol{v}=\left(v_{k}: k \in \mathrm{K}\right)$ is a vector of Lagrange multipliers. The dual function can be expressed as

$$
g(\boldsymbol{v})=\max _{\boldsymbol{\xi} \geq 0, \forall k} L(\boldsymbol{\xi}, \boldsymbol{v}) .
$$

The dual problem corresponding to the primal problem of Equation (6) can be defined as

$$
\min _{\boldsymbol{v} \geq 0, \forall k} g(\boldsymbol{v})
$$

From the KKT conditions stating that $\partial g(\boldsymbol{v}) / \partial p_{k}=0$, we can derive a quadratic equation for $p_{k}$. Thus, we obtain the optimal unit power price of the $k$-th retailer as follows:

$$
\xi_{k}^{*}=\frac{-Y+\sqrt{Y^{2}-4 X Z}}{2 X}
$$

where $\xi_{k}^{*} \geq 0$,

$$
\left\{\begin{array}{l}
X=A^{2} B, Y=2 A B\left(A \mu_{k}-\eta_{2}\right) \\
Z=\left(A \mu_{k}-\eta_{2}\right)^{2} B+A \eta_{2}-A^{2}\left(\mu_{k}+v_{k}\right), \\
A=\Delta_{p} N_{t r x} \alpha\left(\lambda_{\max }-\lambda_{\min }\right) \\
B=P_{a g g}\left(P_{r e q}^{t x}(\lambda)\right)+\sum_{j \neq k}^{K} \eta_{1} p_{j} .
\end{array}\right.
$$

Equation (18) yields the optimal unit power price for the $k$-th retailer, given the dual variables $(\boldsymbol{\mu}, \boldsymbol{v})$, the power consumption profile of the off-grid BS, the expected BS's power demand, and the power distribution of the other retailers.

\subsection{Algorithm Design and Complexity Analysis}

For a differentiable dual function, the gradient descent method [24] can be utilized for determining the optimal values of the dual variables $(\boldsymbol{\mu}, \boldsymbol{v})$, given by

$$
\begin{aligned}
{\left[\mu_{k}(t+1)\right]^{+} } & =\mu_{k}(t)-\tau_{1}\left\{P_{a g g}\left(P_{r e q}^{t x}(\lambda)\right)-\sum_{k=1}^{K} p_{k}\right\} \\
{\left[v_{k}(t+1)\right]^{+} } & =v_{k}(t)-\tau_{2}\left(P_{k}^{g e n}-p_{k}\right)
\end{aligned}
$$

where $t$ is the time index of iteration and $\tau \in\left\{\tau_{1}, \tau_{2}\right\}$ is a sufficiently small fixed step size. Thus, because the gradient in Equations (13) and (17) satisfies the Lipschitz condition [24], the power distribution $p_{k}$ in Equation (14) and the unit power price $\xi_{k}$ in Equation (18) converge to the optimal values, although the off-grid BS iteratively coordinates the retailers. Here, dual optimality $\mu_{k}^{*}$ at the follower level and dual optimality $v_{k}^{*}$ at the leader level can be achieved efficiently using Algorithm 1 [24]. In general, the complexity of the gradient descent method is $O(1 / \tau)$ [27]. Depending on the step size $\tau$, the number of iterations can be decided. Because dual decomposition guarantees fast convergence speed, the proposed approaches for solving the power distribution problem and the unit power price 
problem are adequate [26]. The proposed power distribution and pricing decision algorithm shows the performance at around $0.12 \mathrm{~s}$ and the average 18 iterations.

\section{Numerical Results}

\subsection{Analysis of RES's and BS's Normalized Profile}

Before analyzing the proposed Stackelberg game based PDS, we calculate the normalized profile for RESs and BS traffic load in Figure 2, which is based on the generation pattern of and the BS traffic pattern during the short time period (i.e., $72 \mathrm{~h}$ ) of areas in Europe [14,15]. While RESs are generated dependent on the climatic fluctuation, BS traffic load is dependent on the incoming population and social issues (e.g., holiday). Nevertheless, BS traffic load shows less fluctuation than RES's generation. Solar PV shows more periodical feature than the generation by wind turbine. If the normalized profile for RESs and BS traffic load is the same, the power supplies for BS operation are enough. In Figure 2, EOs mainly occur in the daytime. However, the occurrence of EOs is low in the night time. According to the analysis of the normalized RES's profile and BS load profile, we classify two operation scenarios for the off-grid BS:

- Sufficient RESs' generation

- Insufficient RESs' generation.

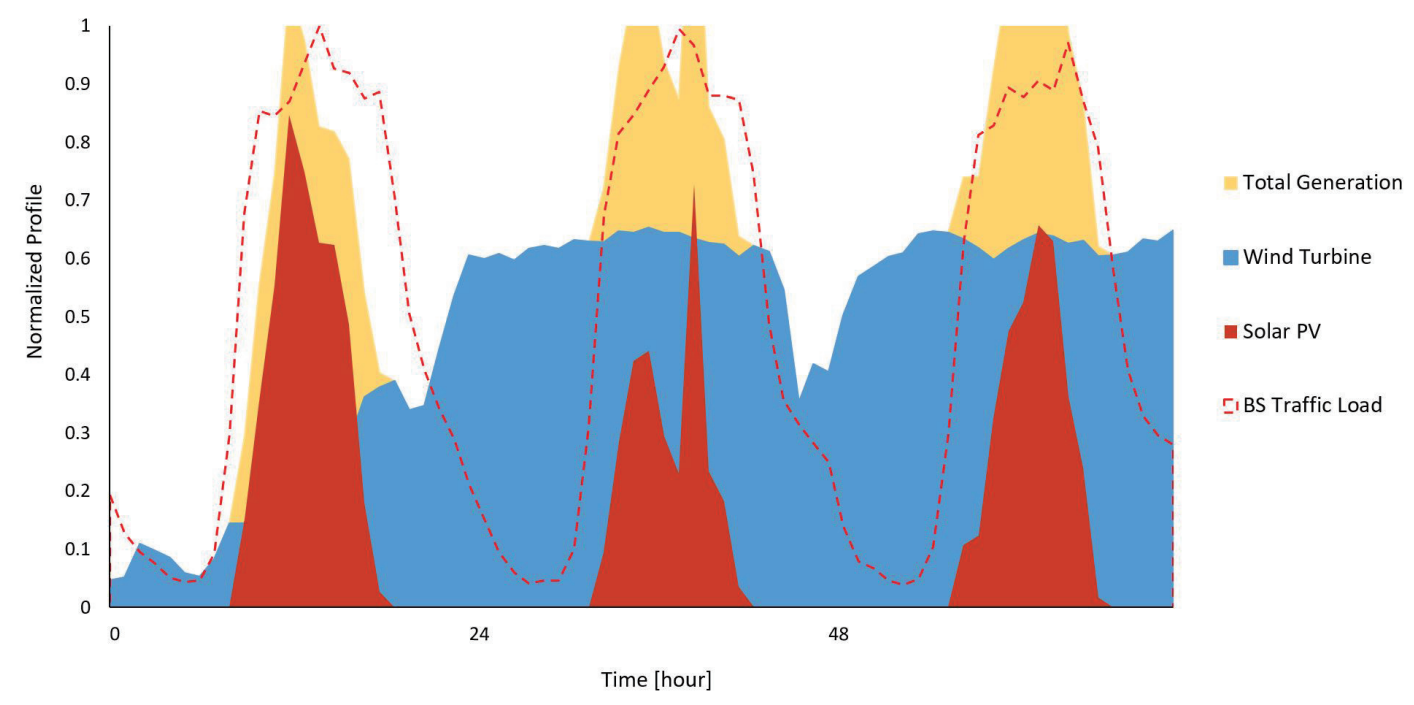

Figure 2. Normalized profile for renewable energy sources (RESs) and base station (BS) traffic load.

The scenario of sufficient RESs' generation describes that off-grid BS operation is sustainable without the support of a battery solution. That is, required off-grid BS operational power is equal to aggregated power distribution. Next, the scenario of insufficient RESs' generation describes that the support of the battery solution is required for the reliable off-grid BS operation. This scenario means that the required off-grid BS operational power is less than the aggregated power distribution. Here, we do not consider electricity of the battery storage as substitute resource of power distribution.

\subsection{Case 1: Sufficient RESs' Generation}

We evaluated the performance of the proposed PDS for a single off-grid BS and two retailers, which provide wind-onshore and solar PV, respectively. First, the unit power price of each retailer is shared to the off-grid BS. However, the information on the retailer's power generation is not shared to the off-grid BS. We consider weighted average levelized costs of electricity (LOCE) for OECD countries in 2014, where the initial unit power prices for wind-onshore and solar PV were set as 
0.15 and $0.3 \$ / \mathrm{kW}$, respectively [28]. The BS's power consumption complies with the macro BS's profile in [23]. We report the detailed numerical results for illustrating the behavior of the proposed PDS. We consider that the proposed PDS distributes power to the off-grid BS via retailers with heterogeneous RES profiles. The mean arrival rate $\lambda$ for the off-grid BS in each fixed time interval is uniformly distributed over $\left[\lambda_{\min }, \lambda_{\max }\right]$. The main parameters for numerical analysis are defined as follows: $P_{1}^{\text {gen }}=0.7 \mathrm{~kW}, P_{2}^{\text {gen }}=0.5 \mathrm{~kW}, \lambda \sim[0,20], \eta_{1}=4, \eta_{2}=0.23$.

Figure 3 shows the comparison of optimal power distribution between the proposed PDS and the EO-PDS. Because the EO-PDS does not consider any unit power price, we assume that the unit power price for the EO-PDS is equally set. The optimal power distribution for the proposed PDS is more balanced that for the EO-PDS, because the EO-PDS ignores the unit power price and considers only the amount of generated power. The optimal unit power prices of retailer 1 and 2 are obtained as 0.5717 and $0.1353 \$ / \mathrm{kW}$, respectively.

Figure 4 shows the optimal power distribution and the optimal unit power price for the mean arrival rate $\lambda$. According to the increasing BS's power demand based on the mean arrival rate $\lambda$, the optimal power distribution and the optimal unit power price increase owing to the hierarchical decision-making mechanism of the underlying Stackelberg game.

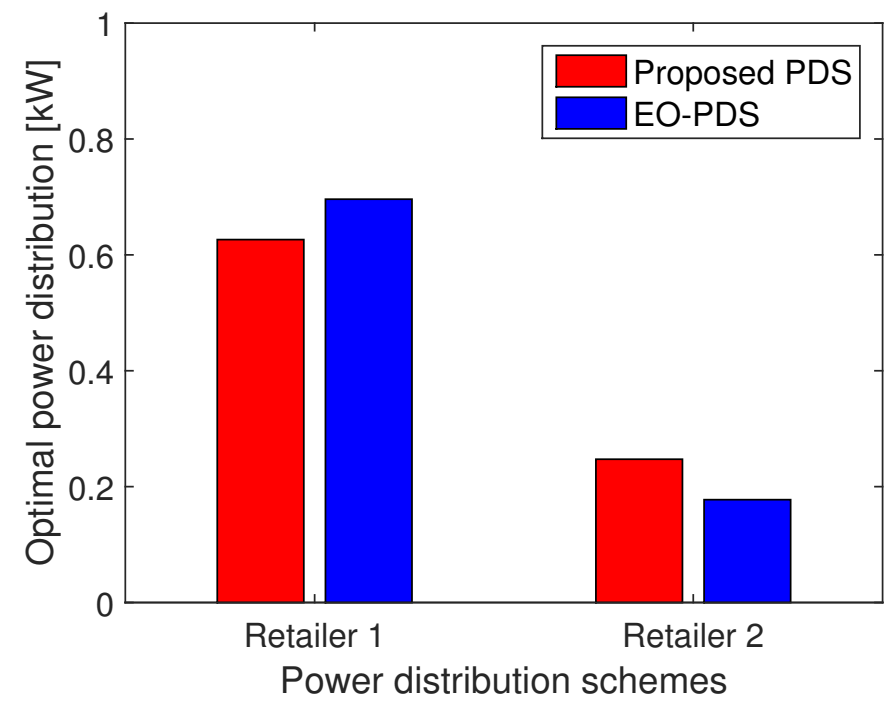

Figure 3. Case 1: comparison of optimal power distribution between the proposed power distribution scheme (PDS) and energy outages-aware (EO)-PDS.

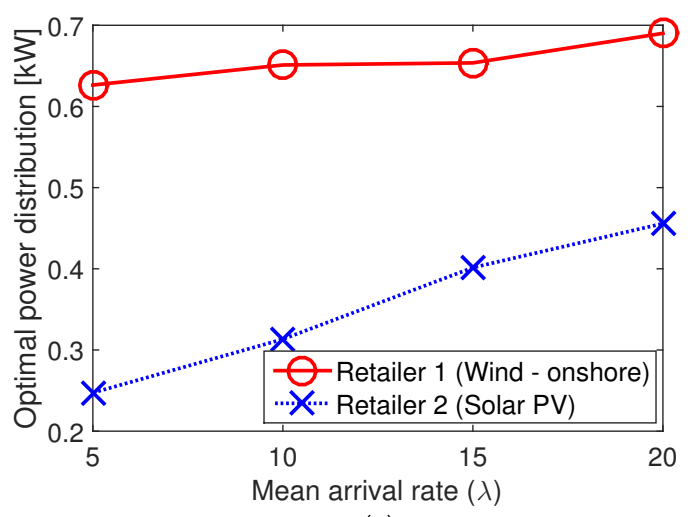

(a)

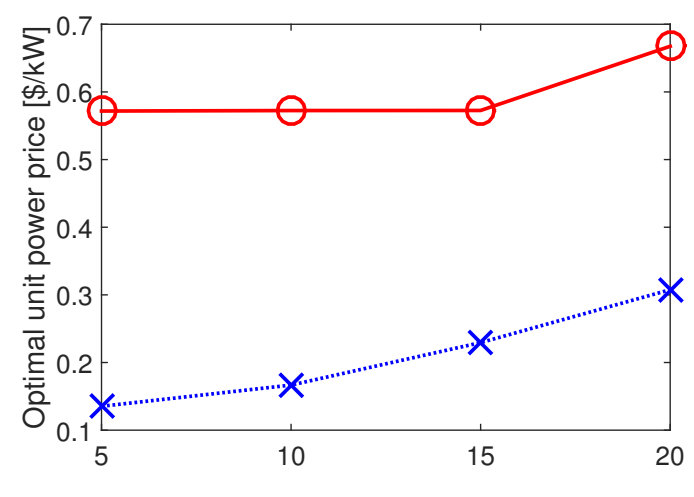

(b)

Figure 4. Case 1: (a) optimal power distribution and (b) optimal unit power price for the mean arrival rate $\lambda$. 
Finally, the utilities of the retailers and the off-grid BS for the mean arrival rate $\lambda$ are shown in Figure 5. According to the optimal strategies of the retailers and the off-grid BS for increasing $\lambda$, the retailers' revenues improve proportional to the increase of power distribution. However, the off-grid BS's utility is slightly reduced due to the increased power cost.

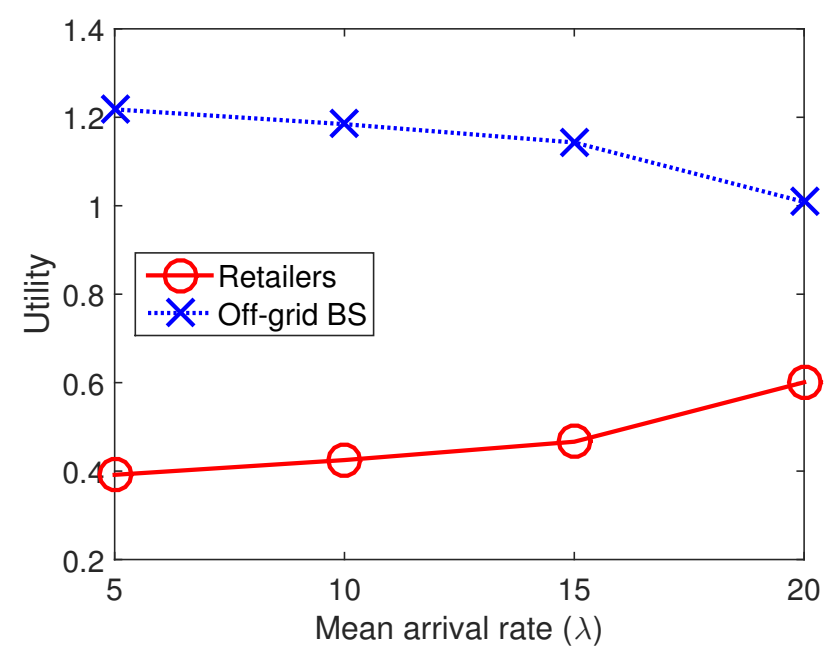

Figure 5. Case 1: retailers' and off-grid BS's utilities for the mean arrival rate $\lambda$.

\subsection{Case 2: Insufficient RESs' Generation}

In this subsection, we consider insufficient RESs' generation as well as the occurrence of EOs. Thus, the modified parameters for numerical analysis are defined as follows: $P_{1}^{g e n}=0.2 \mathrm{~kW}$, $P_{2}^{g e n}=0.5 \mathrm{~kW}$. Accordingly, the off-grid BS should use its own battery storage for the mean arrival rate $\lambda$.

Figure 6 shows the off-grid BS's own battery usage and amount of EOs for the mean arrival rate $\lambda$. Here, since retailers' generation is not changed (i.e., is not increased), battery should support insufficient power distribution. Thus, for increasing the mean arrival rate $\lambda$, the amount used in the off-grid BS's battery as well as EOs also increase.

Figure 7 shows EO probability for mean arrival rate $\lambda$. According to Figure 6 even though the total RESs' generation and the support of the off-grid BS's own battery are used, transmission power to accommodate the increasing mean arrival rate $\lambda$ in the off-grid BS is still insufficient.

Figure 8 shows the optimal power distribution and the optimal unit power price for the mean arrival rate $\lambda$. Under the circumstances that the generation of retailers is not enough, almost the total generation is distributed to the off-grid BS like Figure 8a. Here, the conventional EO-PDS also provides the same amount of power distribution to the off-grid BS. Since EOs increase for the mean arrival rate $\lambda$ in Figures 6 and 7, the optimal unit power price of the retailer increases competitively by the game-theoretic approach. Moreover, compared with Figure $4 \mathrm{~b}$ the optimal unit power price of retailer 1 with large generation is lower than retailer 2 . The proposed PDS can cooperatively guarantee the unit power price for retailer 1 with the insufficient generation. However, retailer 2's revenue is still higher than retailer 1.

Finally, Figure 9 shows the utilities of the retailers and the off-grid BS for the mean arrival rate $\lambda$. The retailers' revenue slightly increases due to the improvement of the optimal unit power price. However, even though the power distribution is not changed for the RESs' generation, compared with Case 1 (sufficient RESs' generation) of Figure 5 the off-grid BS's utility decreases much due to increasing EOs of Figure 7 and increased power cost by the optimal unit power prices of retailers in Figure 8b. 


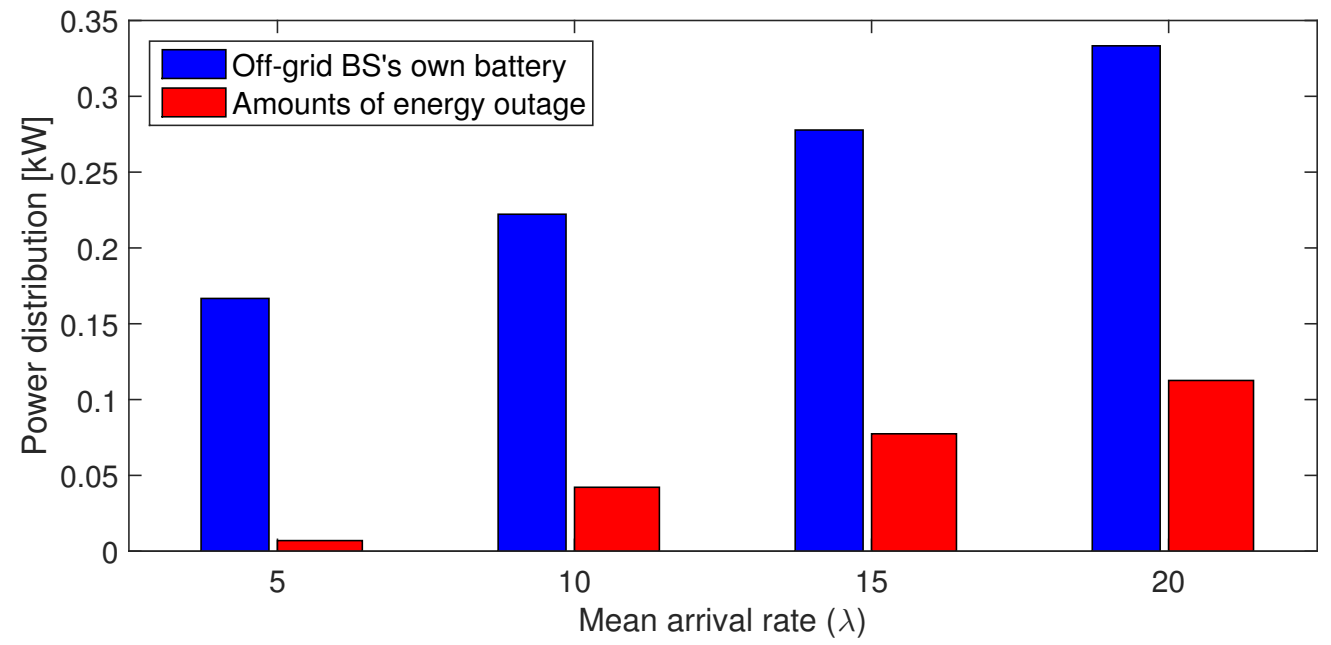

Figure 6. Case 2: off-grid BS's own battery usage and amount of EOs for the mean arrival rate $\lambda$.

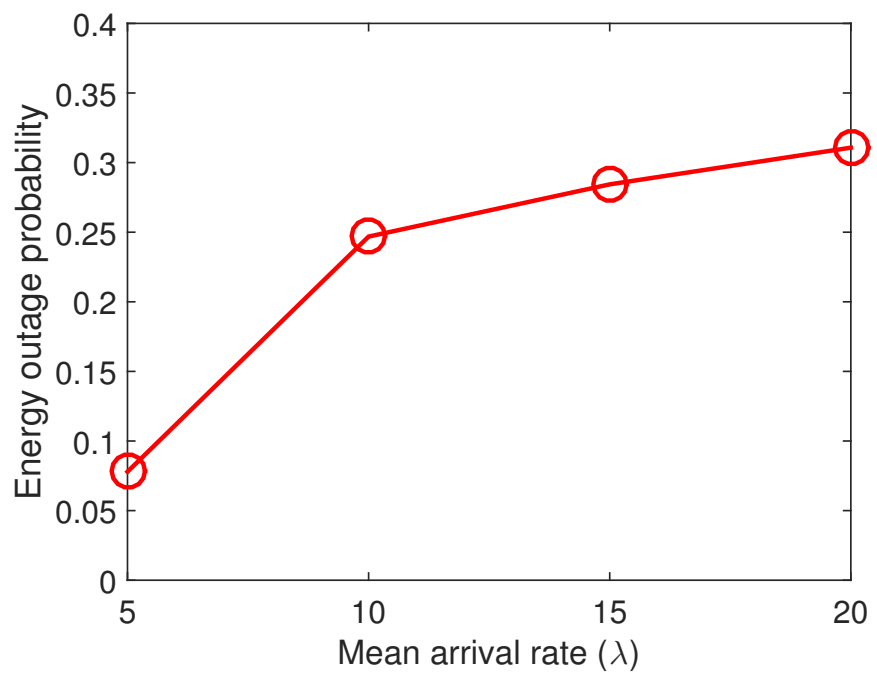

Figure 7. Case 2: energy outage probability for the mean arrival rate $\lambda$.

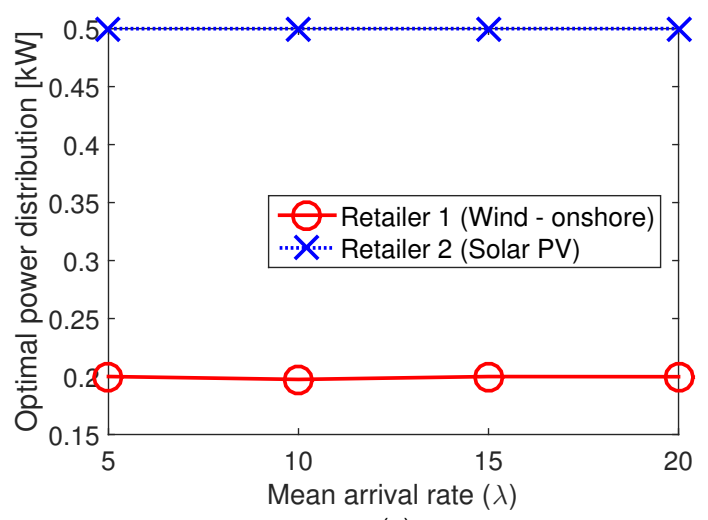

(a)

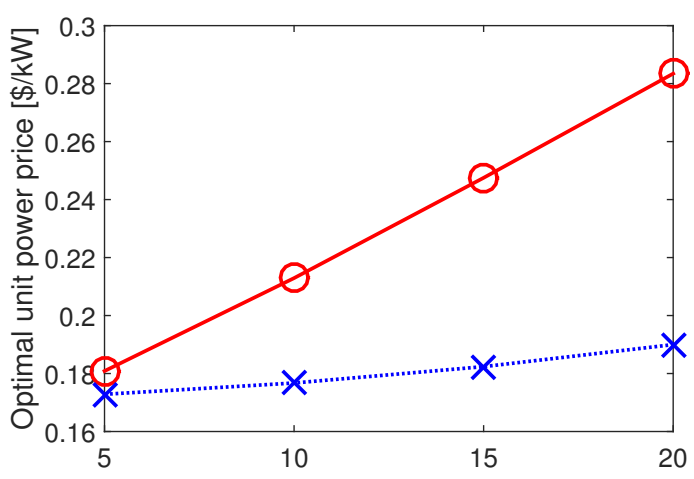

(b)

Figure 8. Case 2: (a) optimal power distribution and (b) optimal unit power price for the mean arrival rate $\lambda$. 


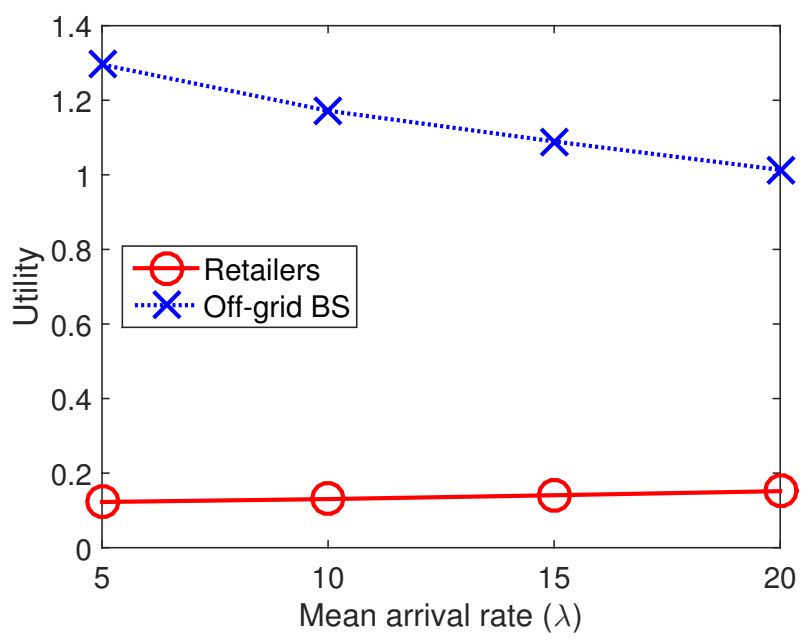

Figure 9. Case 2: retailers' and off-grid BS's utilities for the mean arrival rate $\lambda$.

\section{Conclusions}

In this paper, we have proposed a Stackelberg game based power distribution mechanism for an off-grid BS where multiple retailers compete to maximize their revenues, and where EOs may occur owing to the insufficient generation of RESs. In the proposed mechanism, the iterative interaction between the retailers and the off-grid BS was analyzed by using well-defined utility functions and a closed form expression for the optimal behaviors of players. Finally, using a rigorous game-theoretic analysis, the proposed power distribution showed to converge to a unique equilibrium within an acceptable convergence time, and a maximal payoff was achieved for all participating players in the proposed game model.

Acknowledgments: This work was supported in part by Institute for Information \& communications Technology Promotion (IITP) grant funded by the Korea government (MSIT) [2015-0-00533, Development of TII (Trusted Information Infrastructure) S/W Framework for Realizing Trustworthy IoT Eco-system] and in part by the Korean MSIT (Ministry of Science and ICT), under the National Program for Excellence in SW (2015-0-00932), supervised by the IITP (Institute for Information \& communications Technology Promotion) and in part by the Basic Science Research Program of the National Research Foundation of South Korea under Grant NRF-2018R1C1B6001849.

Author Contributions: Seung Hyun Jeon designed and formulated the Stackelberg game as a first author, and then performed the numerical analysis. Joohyung Lee proposed ideas, validated the game-theoretic approach, and then led the research as a corresponding author. During the manuscript preparation Hong-Shik Park helped to improve the quality of content.

Conflicts of Interest: The authors declare no conflict of interest.

\section{References}

1. Alsharif, M.H.; Kim, J.; Kim, J.H. Green and Sustainable Cellular Base Stations: An Overview and Future Research Directions. Energies 2017, 10, 587.

2. Lee, J.; Guo, J.; Choi, J.K.; Zukerman, M. Distributed Energy Trading in Microgrids: A Game-Theoretic Model and Its Equilibrium Analysis. IEEE Trans. Ind. Electron. 2015, 62, 3524-3533.

3. Park, S.; Lee, J.; Bae, S.; Hwang, G.; Choi, J.K. Contribution-Based Energy-Trading Mechanism in Microgrids for Future Smart Grid: A Game Theoretic Approach. IEEE Trans. Ind. Electron. 2016, 63, 4255-4265.

4. Dhillon, H.S.; Li, Y.; Nuggehalli, P.; Pi, Z.; Andrews, J.G. Fundamentals of Heterogeneous Cellular Networks with Energy Harvesting. IEEE Trans. Wirel. Commun. 2014, 13, 2782-2797.

5. Ulukus, S.; Yener, A.; Erkip, E.; Simeone, O.; Zorzi, M.; Grover, P.; Huang, K. Energy Harvesting Wireless Communications: A Review of Recent Advances. IEEE J. Sel. Areas Commun. 2015, 33, 360-381.

6. Jeon, S.H.; Lee, J.; Choi, J.K. Energy Outage-Aware Power Distribution Scheme for Off-Grid Base Station Operation. IEEE Commun. Lett. 2017, 21, 1401-1404. 
7. Ghazzai, H.; Yaacoub, E.; Alouini, M.S.; Abu-Dayya, A. Optimized Smart Grid Energy Procurement for LTE Networks Using Evolutionary Algorithms. IEEE Trans. Veh. Technol. 2014, 63, 4508-4519.

8. Li, D.; Saad, W.; Guvenc, I.; Mehbodniya, A.; Adachi, F. Decentralized Energy Allocation for Wireless Networks With Renewable Energy Powered Base Stations. IEEE Trans. Commun. 2015, 63, 2126-2142.

9. Deshmukh, M.; Deshmukh, S. Modeling of hybrid renewable energy systems. Renew. Sust. Energ. Rev. 2008, 12, 235-249.

10. Martin, R. Nearly 27,000 Off-Grid Remote Sensing Power Systems Using Renewable or Alternative Energy Will be Deployed Annually by 2020. In Off-Grid Power for Remote Sensing Applications; Navigant Research: Chicago, IL, USA, 2017.

11. Chamola, V.; Sikdar, B. Solar powered cellular base stations: Current scenario, issues and proposed solutions. IEEE Commun. Mag. 2016, 54, 108-114.

12. Aris, A.M.; Shabani, B. Sustainable Power Supply Solutions for Off-Grid Base Stations. Energies 2015, 8, 10904-10941.

13. Marsan, M.A.; Bucalo, G.; Caro, A.D.; Meo, M.; Zhang, Y. Towards zero grid electricity networking: Powering BSs with renewable energy sources. In Proceedings of the IEEE International Conference on Communications Workshops (ICC), Budapest, Hungary, 9-13 June 2013; pp. 596-601.

14. Jeon, S.H.; Lee, J.; Choi, J.K. A Distributed Power Allocation Scheme for Base Stations Powered by Retailers with Heterogeneous Renewable Energy Sources. ETRI J. 2016, 38, 746-756.

15. Mao, Y.; Luo, Y.; Zhang, J.; Letaief, K.B. Energy harvesting small cell networks: feasibility, deployment, and operation. IEEE Commun. Mag. 2015, 53, 94-101.

16. Alsharif, M.H.; Kim, J. Hybrid Off-Grid SPV/WTG Power System for Remote Cellular Base Stations Towards Green and Sustainable Cellular Networks in South Korea. Energies 2017, 10, 9.

17. Chamola, V.; Sikdar, B. Power Outage Estimation and Resource Dimensioning for Solar Powered Cellular Base Stations. IEEE Trans. Commun. 2016, 64, 5278-5289.

18. Parzysz, F.; Renzo, M.D.; Verikoukis, C. Power-Availability-Aware Cell Association for Energy-Harvesting Small-Cell Base Stations. IEEE Trans. Wirel. Commun. 2017, 16, 2409-2422.

19. Chamola, V.; Krishnamachari, B.; Sikdar, B. Green Energy and Delay Aware Downlink Power Control and User Association for Off-Grid Solar-Powered Base Stations. IEEE Syst. J. 2017, PP, 1-12.

20. Zhang, H.; Jiang, C.; Beaulieu, N.C.; Chu, X.; Wang, X.; Quek, T.Q.S. Resource Allocation for Cognitive Small Cell Networks: A Cooperative Bargaining Game Theoretic Approach. IEEE Trans. Wirel. Commun. 2015, 14, 3481-3493.

21. Zhang, H.; Du, J.; Cheng, J.; Long, K.; Leung, V.C.M. Incomplete CSI Based Resource Optimization in SWIPT Enabled Heterogeneous Networks: A Non-Cooperative Game Theoretic Approach. IEEE Trans. Wirel. Commun. 2018, 17, 1882-1892.

22. Son, K.; Kim, H.; Yi, Y.; Krishnamachari, B. Base Station Operation and User Association Mechanisms for Energy-Delay Tradeoffs in Green Cellular Networks. IEEE J. Sel. Areas Commun. 2011, 29, 1525-1536.

23. Auer, G.; Giannini, V.; Desset, C.; Godor, I.; Skillermark, P.; Olsson, M.; Imran, M.A.; Sabella, D.; Gonzalez, M.J.; Blume, O.; et al. How much energy is needed to run a wireless network? IEEE Wirel. Commun. Mag. 2011, 18, 40-49.

24. Boyd, S.; Vandenberghe, L. Convex Optimization; Cambridge University Press: Cambridge, UK, 2004.

25. Wang, Y.; Wang, X.; Wang, L. Low-Complexity Stackelberg Game Approach for Energy-Efficient Resource Allocation in Heterogeneous Networks. IEEE Commun. Lett. 2014, 18, 2011-2014.

26. Palomar, D.P.; Chiang, M. A tutorial on decomposition methods for network utility maximization. IEEE J. Sel. Areas Commun. 2006, 24, 1439-1451.

27. Gordon, G.; Tibshirani, R. Lecture 5: Gradient Desent Revisited. In 10-725 Optimization; Carnegie Mellon University: Pittsburgh, PA, USA, 2012.

28. IRENA. Figure 2.7: The levelised cost of electricity by region and technology and their weighted average, 2013/2014. In Renewable Power Generation Costs in 2014; International Renewable Energy Agency (IRENA): Abu Dhabi, UAE, 2015.

(C) 2018 by the authors. Licensee MDPI, Basel, Switzerland. This article is an open access article distributed under the terms and conditions of the Creative Commons Attribution (CC BY) license (http:// creativecommons.org/licenses/by/4.0/). 